\title{
Effects of kefir or milk supplementation on zonulin in overweight subjects
}

\author{
Z. Jenko Pražnikar, ${ }^{1}$ S. Kenig, ${ }^{1}$ T. Vardjan, ${ }^{2}$ M. Černelič Bizjak, ${ }^{1}$ and A. Petelin ${ }^{1 *}$ \\ ${ }^{1}$ Faculty of Health Sciences, University of Primorska, Polje 42, SI-6310 Izola, Slovenia \\ ${ }^{2}$ Kele \& Kele, d.o.o., Laze 22/a, SI-1370 Logatec, Slovenia
}

\section{ABSTRACT}

Increased intestinal permeability has been shown to be involved in several diseases associated with lowgrade chronic inflammation, including obesity and metabolic syndrome. In the last decade, growing evidence shows the beneficial effects of probiotic-containing food supplementation on these conditions. In this crossover intervention study on 28 asymptomatic overweight adults, we tested the effects of a 3-wk kefir supplementation compared with a 3 -wk milk supplementation on serum zonulin levels. The effects on serum glucose, triacylglycerols, low-density lipoproteins, high-density lipoproteins, total cholesterol, markers of inflammation (C-reactive protein and adiponectin), anthropometric variables, mood, and appetite were also determined. Kefir supplementation resulted in a greater improvement of serum zonulin levels $\left(F=6.812, \eta^{2}=0.275\right)$, whereas a significant yet similar improvement in lipid profile and serum glucose levels was found in both supplementations. Positive mood was slightly but significantly enhanced with kefir supplementation, and reduced with milk supplementation. The C-reactive protein, adiponectin, and appetite were unaffected. In conclusion, supplementation with both dairy products had health beneficial effects, but only kefir showed an effect on the intestinal barrier dysfunction marker.

Key words: kefir, low-grade chronic inflammation, intestinal permeability, overweight

\section{INTRODUCTION}

Today, we are facing a worldwide epidemic of metabolic syndrome and obesity. Metabolic syndrome is a cluster of interrelated risk factors including obesity, especially visceral obesity, impaired glucose tolerance, insulin resistance, hyperlipidemia, and hypertension (Alberti et al., 2009). These factors increases the risk of diabetes, liver dysfunction, neurodegenerative disor-

Received October 3, 2019.

Accepted January 20, 2020

*Corresponding author: ana.petelin@upr.si ders, and cardiovascular diseases (Zimmet and Alberti, 1997; Scherer and Hill, 2016), which are the leading cause of death globally [World Health Organization; https://www.who.int/news-room/fact-sheets/detail/ cardiovascular-diseases-(cvds)].

It has been shown that the development of metabolic syndrome and related chronic disorders may be explained by chronic low-grade systemic inflammation with progressive immune cell infiltration into adipose tissue, which is recognized in obesity (Ouchi et al., 2011). Recent studies suggest that gut microbiota dysbiosis may contribute importantly to such a state (Everard and Cani, 2013; Festi et al., 2014; Boulangé et al., 2016; Gérard, 2016) through several mechanisms, including impairment of intestinal wall integrity, harvest of energy, or a disturbed mucosal immune system (Arrieta et al., 2006; Chassaing and Gewirtz, 2014). The upregulation of zonulin, which regulates intestinal permeability by modulating intracellular tight junctions and is commonly used as a serum marker of compromised intestinal wall integrity (Wang et al., 2000), has been associated with these conditions (Sapone et al., 2006; Moreno-Navarrete et al., 2012; Zak-Gołąb et al., 2013; Jayashree et al., 2014; Genser et al., 2018).

In recent years, interest has increased in using probiotics and prebiotics as a complementary or adjuvant therapy in the treatment of certain diseases and mood disorders (Ostadrahimi et al., 2015; Rios et al., 2017; Ejtahed et al., 2019). Kefir, a fermented dairy product produced through the symbiotic fermentation of milk by lactic acid bacteria and yeast, is of interest due to its suggested beneficial properties on health, including control of plasma glucose, and antihypertensive, antiinflammatory, anti-allergenic, antioxidant, antibacterial, and hypocholesterolaemic effects (reviewed in Rosa et al., 2017; reviewed in Pimenta et al., 2018). Milk supplementation also had beneficial effects on body fat mass (Li et al., 2018) and an inverse association with insulin resistance syndrome was observed (Pereira et al., 2002).

Manipulation of gut microbiota homeostasis with the consumption of probiotics may be a profitable strategy to prevent or attenuate several metabolic complica- 
Table 1. Nutritional values of kefir and milk

\begin{tabular}{lcc}
\hline Nutritional value $(100 \mathrm{~g})$ & Kefir & Milk \\
\hline Energy value (kJ, kcal) & 57,239 & 64,266 \\
Fat content (g) & 3.2 & 3.5 \\
SFA (g) & 2.4 & 2.3 \\
Carbohydrates (g) & 3.9 & 4.7 \\
Sugars (g) & 3.9 & 4.7 \\
Proteins (g) & 3.2 & 3.3 \\
Salt (g) & 0.1 & 0.12 \\
Calcium (g) & 0.12 & 0.12 \\
\hline
\end{tabular}

tions (Pimenta et al., 2018). Dietary modifications are known to be most efficient in patients at the early stage of the development of metabolic syndrome components, for example, in mild hypercholesterolemia, mildly increased glucose, or overweight, when these are still likely reversible. Therefore, the aim of our study was to investigate the effects of traditional kefir in comparison to milk intake in asymptomatic overweight adults on serum zonulin levels. In addition, we followed inflammatory markers, lipid profile, serum glucose levels, anthropometric variables, and mood.

\section{MATERIALS AND METHODS}

\section{Kefir and Milk Characteristics}

The kefir used in our study was produced by Kele \& Kele d. o. o., Logatec, Slovenia. The exact lactobacilli and yeast composition was shown to be very stable over time (Vardjan et al., 2013; Vardjan et al., 2018) and was reported in detail along with physical and chemical properties, such as water and DM content and $\mathrm{pH}$ value
(Vardjan et al., 2018). Briefly, close to $90 \%$ of bacterial isolates were genus Lactobacillus (Lactobacillus parakefiri, Lactobacillus kefiri, and Lactobacillus kefiranofaciens ssp. kefirgranum), and the remaining isolates were cocci. Kluyveromyces marxianus, Kazachstania exigua, and Rhodosporidium kratochvilovae were the predominant yeast species. The water content was around $80 \%$, $\mathrm{pH}$ was 4.03, and the exact nutritional value is reported in Table 1. Milk produced by Ljubljanske mlekarne (Ljubljana, Slovenia) with the same fat content was used, and its nutritional values are in Table 1.

\section{Study Design}

This study was conducted at the University of Primorska, Faculty of Health Sciences, from April to June in 2018. The crossover intervention study lasted $8 \mathrm{wk}$ and was organized into 2 different phases. A blinded investigator randomly allocated the participant to 1 of the 2 groups by a computer program. Fourteen participants consumed $300 \mathrm{~mL}$ of kefir per day in the first 21-d intervention period, whereas 14 participants consumed $300 \mathrm{~mL}$ of milk per day first. During the second intervention period, participants consumed the alternate drink. Both kefir and milk were packed in 300 -g pots. The study was randomized but unblinded for participants and clinical investigators. The basic composition of both products was similar (Table 1). The two 21-d intervention periods were separated by a 7-d washout period, during which the participants abstained from eating dairy products. Participants were asked to consume the milk or kefir drink at breakfast time daily. At the end of each intervention or washout

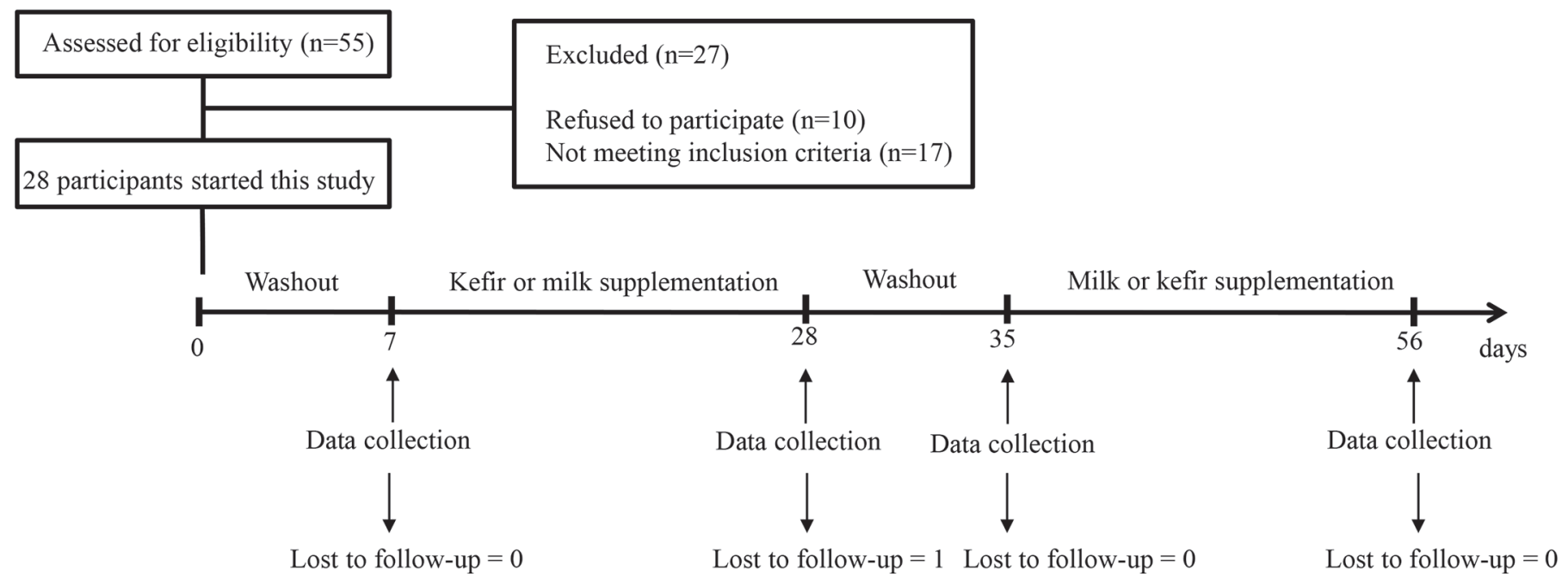

Figure 1. Flow diagram of participants throughout the study. 
period, anthropometric measurements were performed and serum samples were collected (Figure 1). Compliance to the treatment was monitored by interviewing the participants during visits.

Throughout the study, the participants were encouraged to avoid any alterations to their normal diet or exercise but were not allowed to consume additional prebiotics or probiotics in any form, or drink milk in the kefir intervention period. A 24 -h recall was administered after the washout period and 3 times during consumption of the dairy products to assess the food intake during the study. An expert dietitian collected an in-person list of all foods and beverages, using food models and standardized version of 5-pass method developed by the US Department of Agriculture. Open Platform for Clinical Nutrition, accessible through the website http://opkp.si/, was used to calculate energy and dietary intake.

\section{Study Participants}

Fifty-five Caucasian healthy overweight participants aged between 30 and 60 years were screened for enrolment in the study. Eligible participants had to meet the following criteria: age between 30 and $60 \mathrm{yr}$, body mass index (BMI) between 25 to $29.9 \mathrm{~kg} / \mathrm{m}^{2}$, no body mass change exceeding $3 \%$ within the last $3 \mathrm{mo}$, no acute or chronic diseases, no gastrointestinal diseases or endocrine disorders, no drug use for lipid disorders or anti-inflammatory drugs, no nutritional supplements, antibiotics, not pregnant, and not lactating. The male and female volunteers were recruited through advertisement posted in Internet forums, sent via email, and in local newspapers. Seventeen participants did not meet the inclusion criteria, and 10 participants declined to participate. A total of 28 subjects (13 males and 15 females) fulfilled the eligibility criteria to participate. Twenty-seven participants completed the study, one participant dropped out because of chickenpox (Figure 1). The study protocol was approved by the Slovenian National Medical Ethics Committee (No. 0120-206/2018/4). All participants signed their written informed consent before the beginning of the intervention.

\section{Sample Size}

Sample size was calculated based on Zak-Gołąb et al. (2013) study. Assuming type I error probability $\alpha$ $=0.05,26$ participants were estimated to provide $80 \%$ power with a 2 -sided test to detect a $15 \%$ decrease in serum zonulin levels after kefir intervention. In addition, assuming $10 \%$ dropout during the study, we increased the number of participants to 29 .

\section{Outcome Variables}

The primary objective of this crossover interventional study was to investigate the effects of traditional kefir in comparison to milk intake in asymptomatic overweight adults on serum zonulin levels. In addition, we followed inflammatory marker C-reactive protein (CRP) and anti-inflammatory adiponectin, triacylglycerols (TAG), total cholesterol, low-density lipoprotein (LDL), high-density lipoprotein (HDL), serum glucose levels, anthropometric variables [weight, fat free mass, fat (\%), muscle mass, visceral fat], and mood.

\section{Anthropometric Measurements}

Anthropometric variables were measured following an overnight fast between 7 a.m. and 8 a.m. in standardized conditions by the same examiner. Body weight was measured in light clothing without shoes to the nearest $0.1 \mathrm{~kg}$ and height to the nearest $0.1 \mathrm{~cm}$, using a Leicester Height Measure (Invicta Plastics Limited, Oadby, UK). The BMI was calculated as weight $(\mathrm{kg})$ divided by height $(\mathrm{m})$ squared. Body composition (total percentage of body fat and fat-free mass) was assessed using bioelectrical impedance analysis by Tanita MC-980MA (Maeno-cho, Japan) and dedicated software (GMON Pro-Tanita).

\section{Blood Measurements}

Blood samples were taken on d 7, 28, 35, and 56. The 8 -mL samples of venous blood were collected from the median cubital vein in the morning following an overnight fast in vacuum test tubes. Serum aliquots were stored at $-80^{\circ} \mathrm{C}$. Serum concentrations of glucose, TAG, total cholesterol, LDL, HDL, and CRP were measured with Cobass c111 analyzer (Roche, Basel, Switzerland). In addition, serum concentrations of adiponectin and zonulin were measured in duplicate on a microplate reader (Tecan, Männedorf, Switzerland) using human ELISA Kits for adiponectin (BioVendor, Prague, Czech Republic) and zonulin (MyBioSource Inc., San Diego, CA). Assay sensitivity was $10 \mathrm{pg} / \mathrm{mL}$ for adiponectin and $0.5 \mathrm{ng} / \mathrm{mL}$ for zonulin.

\section{Mood Measure}

Mood was measured using the positive and negative affect schedule questionnaire (Watson et al., 1988), which has acceptably high $\alpha$ reliabilities, ranging from 0.86 to 0.90 . It is a 20 -item scale. Ten items assessed positive affect (e.g., excited, active, enthusiastic), and 10 items measured negative affect (e.g., irritable, distressed, nervous); high positive affect is a state of high 
Table 2. Baseline characteristics of participants ${ }^{1}$

\begin{tabular}{lc}
\hline Item & Mean $\pm \mathrm{SD}$ \\
\hline Characteristic $^{2}$ & $15 / 13(53.6 / 46.4)$ \\
Sex [female/male (\%)] & $45.8 \pm 8.4$ \\
Age (yr) & $29.1 \pm 4.6$ \\
BMI (kg/m $\left.{ }^{2}\right)$ & $89.7 \pm 19.6$ \\
Weight $(\mathrm{kg})$ & $6.5 \pm 1.1$ \\
Biochemical variable & $1.3 \pm 0.6$ \\
Fasting glucose (mmol/L) & $5.8 \pm 1.8$ \\
TAG (mmol/L) & $1.7 \pm 0.4$ \\
TC (mmol/L) & $3.9 \pm 1.7$ \\
HDL cholesterol (mmol/L) & $1.99 \pm 1.19(1.54 ; 0.4-4.54)$ \\
LDL cholesterol (mmol/L) & \\
CRP ${ }^{4}(\mathrm{mg} / \mathrm{L})$ &
\end{tabular}

${ }^{1}$ Values are presented as means \pm SD.

${ }^{2} \mathrm{BMI}=$ body mass index.

${ }^{3} \mathrm{TC}=$ total cholesterol; $\mathrm{CRP}=\mathrm{C}$-reactive protein; $\mathrm{HDL}=$ highdensity lipoproteins; $\mathrm{LDL}=$ low-density lipoproteins; $\mathrm{TAG}=$ triacylglycerols.

${ }^{4} \mathrm{CRP}$ value is presented as mean $\pm \mathrm{SD}$ and as median (minimum and maximum) values.

energy, full concentration, and pleasurable engagement, whereas low positive affect is characterized by sadness and lethargy. Subjects were instructed to respond to the positive and negative affect schedule questionnaire on the basis of how they felt at that moment and indicate "to what extent do you feel this way right now?" Their answers ranged from 5 (extremely) to 1 (very slightly or not at all).

\section{Appetite Measure}

The visual analog scale for appetite measurement, satiety, and desire to eat consisted of 4 scales $(100 \mathrm{~mm}$ lines) anchoring extreme appetite perceptions on both ends of each line (e.g., not at all hungry to very hungry; Sepple and Read, 1989). Subjects were requested to make a vertical mark on a line that best matched how they were feeling in the last few days. This scale was anchored at 0 (not at all hungry) to 7 (very hungry). Each score was determined by measuring the distance from the left side of the line to the mark. Average subjective appetite value was calculated as the average of the measured distances for all visual scales.

\section{Statistics}

We applied per protocol analysis. Throughout the study, only one participant dropped out and all participants reported to be compliant to the intervention. Statistical analysis was performed using SPSS version 23.0 (IBM Corp., Armonk, NY). The normality of variables was tested by the Shapiro-Wilk test. Before statistical analysis, obtained data that were not normally distributed (CRP and zonulin levels) were logarithmically transformed to approximate a normal distribution for subsequent analysis. Normally distributed data are presented as the mean value with standard deviation or percentage. In addition, CRP and zonulin values are presented as the mean value with standard deviation and as median (minimum and maximum) values. The effects of the interventions within each group were analyzed using a paired sample $t$-test, whereas comparison of mean changes between the 2 groups was analyzed using an independent $t$-test. Moreover, the effects of the interventions were analyzed using an univariate analysis of covariance (ANCOVA) with the change at wk 3 from baseline as the dependent variable, adjusted to the corresponding values at baseline, and stratified for several variables (age, sex, energy intake, and anthropometric and biochemical variables at baseline). $P$-values $<0.05$ were considered statistically significant.

\section{RESULTS}

\section{Baseline Characteristics of Participants and Compliance}

Table 2 illustrates the baseline characteristics of participants. The study population was $46.4 \%$ male and $53.6 \%$ female with a mean age of $45.8 \pm 8.4 \mathrm{yr}$. Initial BMI and weight before kefir or milk supplementation were $29.1 \pm 4.6 \mathrm{~kg} / \mathrm{m}^{2}$ and $89.7 \pm 19.6 \mathrm{~kg}$, respectively. Most of the participants had elevated serum levels of glucose and mild to moderate hypercholesterolemia; the mean glucose, TAG, total cholesterol, HDL cholesterol, and LDL cholesterol were $6.5 \pm 1.1,1.3 \pm 0.6,5.8 \pm$ $1.8,1.7 \pm 0.4$, and $3.9 \pm 1.7 \mathrm{mmol} / \mathrm{L}$, respectively. The mean CRP was $1.99 \pm 1.19 \mathrm{mg} / \mathrm{L}$.

All participants were compliant with the study. Few participants reported gas problems, feelings of bloating, having loose stools, diarrhea, or cramping. Gastrointestinal disturbances were reported during both intervention periods. Apart from that, both products were well tolerated. Energy intake and the macronutrient composition of the diets did not change during either intervention period (Table 3 ).

\section{Effects of Kefir and Milk Intake on Anthropometric and Biochemical Variables}

The effects of kefir and milk consumption on anthropometric data, glucose, and lipid metabolism were evaluated (Table 4). In the present study, the paired $t$ test revealed no effects of kefir or milk supplementation on anthropometric variables. For BW, fat-free mass, percentage of fat, muscle mass, and visceral fat values, 
Table 3. Characteristics of diet during kefir and milk supplementation ${ }^{1}$

\begin{tabular}{lccc}
\hline Item & Kefir intake & Milk intake & $P$-value \\
\hline Energy value (kcal/d) & $1,852 \pm 704$ & $1,906 \pm 581$ & 0.826 \\
Proteins $(\mathrm{g} / \mathrm{d})$ & $81 \pm 30$ & $91 \pm 32$ & 0.381 \\
Carbohydrates (g/d) & $187 \pm 82$ & $206 \pm 81$ & 0.554 \\
Fat $(\mathrm{g} / \mathrm{d})$ & $84 \pm 42$ & $77 \pm 31$ & 0.600 \\
Fiber $(\mathrm{g} / \mathrm{d})$ & $18.4 \pm 13.6$ & $16.9 \pm 15.7$ & 0.806 \\
Ca $(\mathrm{mg} / \mathrm{d})$ & $951 \pm 229$ & $922 \pm 615$ & 0.869 \\
\hline
\end{tabular}

${ }^{1}$ Values are presented as means $\pm \mathrm{SD}$.

${ }^{2} P$-value denotes differences between the 2 groups using an independent $t$-test.

no significant changes were observed within the groups in either of the 2 interventions. Similarly, using the ANCOVA model with adjustment for the corresponding values at baseline and stratified for several variables, no significant changes were found.

In the analysis of biochemical variables with a paired $t$-test, we found a statistically significant decrease in total cholesterol, LDL cholesterol, and glucose after milk supplementation, whereas kefir supplementation resulted in decreased glucose and HDL concentrations. After adjustments for the described variables (ANCOVA model $)$, the reductions in total cholesterol $(P<0.001$, $F=19.480, \eta^{2}=0.520$ in kefir; $P=0.007, F=19.663$, $\eta^{2}=0.663$ in milk supplementation), LDL cholesterol $\left(P<0.001, F=18.317, \eta^{2}=0.504\right.$ in kefir; $P<0.001$, $F=39.895, \eta^{2}=0.799$ in milk supplementation), and glucose $\left(P<0.001, F=44.690, \eta^{2}=0.680\right.$ in kefir; $P=$ $0.006, F=10.071, \eta^{2}=0.372$ in milk supplementation) were similar and statistically significant after both kefir and milk supplementation. Between the 2 groups, no significant differences were observed in anthropometric and biochemical variables.

\section{Effects of Kefir and Milk Intake on CRP, Adiponectin, and Zonulin}

To test the hypothesized effect of kefir and milk on intestinal permeability and chronic inflammation, we have measured serum levels of CRP, adiponectin, and zonulin. Table 5 shows that after kefir or milk consumption, no significant differences in CRP, adiponectin, and zonulin levels were detected with paired $t$-test. However, ANCOVA test with the change in each variable as the dependent variable, adjustment for baseline values, and stratification for several variables revealed a reduction in zonulin levels $\left(P=0.018, F=6.812, \eta^{2}=0.275\right)$ only after kefir, but not after milk supplementation. Neither of the 2 interventions significantly affected CRP and adiponectin. In addition, no significant differences in inflammatory CRP levels, anti-inflammatory adiponectin, and zonulin levels were found between the 2 groups.

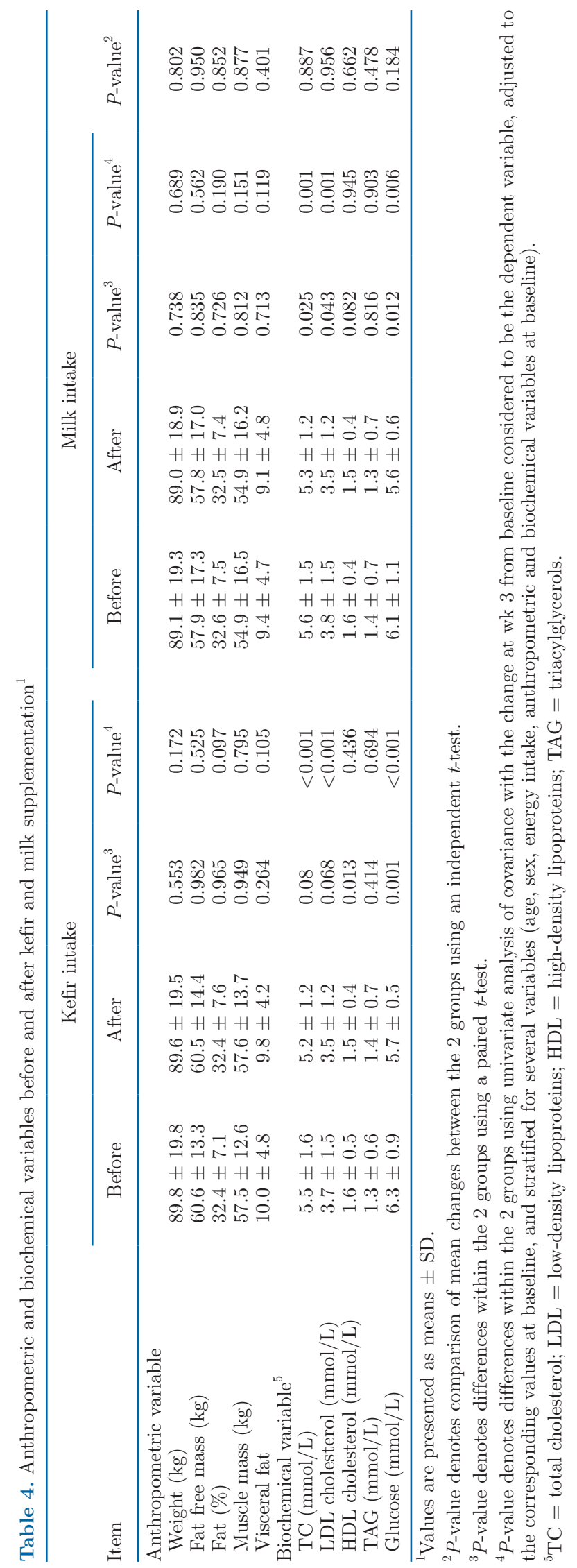




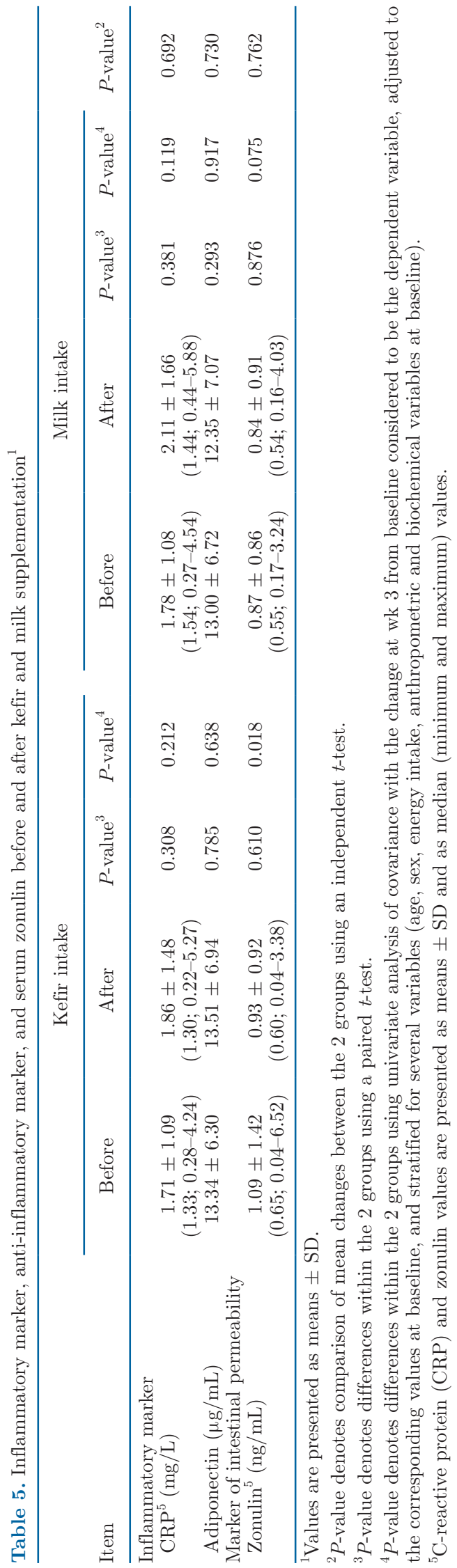

\section{Effects of Kefir and Milk Supplementation on Mood and Appetite}

Table 6 shows that 8 wk of kefir supplementation enhanced $(P=0.033)$ the positive affect or mood, whereas negative mood was slightly but not significantly reduced. On the contrary, we observed reduced $(P$ $=0.032)$ positive mood after milk supplementation. In addition, we detected differences $(P=0.014)$ in positive affect or mood also between the 2 groups. Self-reported appetite perceptions were reduced after 8 wk of kefir supplementation, but not after milk supplementation. However, the differences were not significant.

\section{DISCUSSION}

To our knowledge, this is the first study conducted to establish whether a 3-wk-long kefir or milk supplementation influences serum levels of zonulin in asymptomatic overweight healthy adults. The CRP, adiponectin, lipid profile, serum glucose levels, and anthropometric variables were also investigated. We show that kefir supplementation causes a greater improvement in serum zonulin levels compared with milk supplementation, whereas the improvements in lipid profile and serum glucose levels were similar in both interventions.

Ingestion of probiotics has been found to be a useful therapeutic strategy to prevent or attenuate several diseases, mainly through reestablishment of gut microbiota. This is one potential explanation for the observed zonulin decrease in the intervention with kefir supplementation. Several studies support this hypothesis. Kefir had a positive effect on the abundance of several bacterial species in the gut of rats (Gao et al., 2019), and supplementation with Lactobacillus kefiranofaciens, the most abundant bacteria in our kefir (Vardjan et al., 2013), markedly increased the diversity of gut bacteria in mice (Sun et al., 2019). Bifidobacteria, a group of bacteria also possibly present in kefir has been shown to improve mucosal barrier function (Griffiths et al., 2004). An additional reason why zonulin downregulation could be attributed to the probiotic nature of kefir is the fact that with milk supplementation we did not observe such a difference. A few studies investigating effects of (nonfermented and nonsupplemented) milk reported changes in the relative abundance of gut bacteria but not increased diversity (Norris et al., 2016; Li et al., 2018). Furthermore, a similar outcome as in our study was observed also using different probiotics; in an 8-wk intervention with a probiotic food supplement containing a mixture of 9 bacterial strains (van Hemert et al., 2013), zonulin decreased significantly. The utility of zonulin as a marker is currently under debate because contradicting results regarding its as- 
sociation with irritable bowel syndrome and functional dyspepsia (Talley et al., 2019) were reported. A widely used ELISA kit was found to be nonspecific (Scheffler et al., 2018), which seems to be the most likely reason for the observed discrepancies.

It has been suggested that the impairment of intestinal wall integrity is an important factor contributing to the low-grade chronic inflammation in obesity (Arrieta et al., 2006). In fact, we did not find any significant improvement in CRP and adiponectin levels after kefir or milk supplementation. Increased intestinal permeability involve increasing passage of LPS into the circulation, which subsequently stimulates secretion of inflammatory cytokines by targeting specific tissues and interacting with specific receptors (Cani et al., 2008; Boroni Moreira and de Cássia Gonçalves Alfenas, 2012; Jayashree et al., 2014). The anti-inflammatory potential of kefir has been evaluated in an animal model of asthma, rats with edema, and on the intestinal mucosal immune response in mice (Rodrigues et al., 2005; Vinderola et al., 2005; Lee et al., 2007). Hadisaputro et al. (2012), Rosa et al. (2016), and Kim et al. (2017) found that kefir supplementation improved the balance between proand anti-inflammatory cytokines in an experimental model for diabetes, metabolic syndrome, and obesity. Mechanism explaining the anti-inflammatory properties of kefir may involve direct or indirect action on the gut microbiota. Indirect actions involve the bioactive peptides produced during fermentation, which can activate macrophages and increase phagocytosis and suppression of the Th2 immune response (Adiloğlu et al., 2013). Apart from being a probiotic, kefir contains of $\mathrm{B}$ complex vitamins, vitamins $\mathrm{C}, \mathrm{A}, \mathrm{K}$, and carotene, minerals, and EAA (Otles and Cagindi, 2003), which could all contribute to the reduced inflammation and other positive effects of kefir intake.

Some evidence indicates that kefir and milk supplementation have the ability to improve serum glucose levels in experimental diabetic and metabolic syndrome models and in diabetic humans (Hadisaputro et al., 2012; Ostadrahimi et al., 2015; El-Sayed et al., 2016; Rosa et al., 2016). Here we show that in asymptom- atic overweight healthy adults with mild to moderate hyperglycemia, both kefir or milk consumption significantly reduced serum glucose levels. In line with some previous reports, we also found that both interventions caused a significant decrease in total cholesterol levels and LDL cholesterol levels. Indeed, various studies have concluded that milk and fermented milk products, especially kefir, are able to reduce the serum cholesterol levels in animals and humans (Wang et al., 2009; Soerensen et al., 2014; El-Sayed et al., 2016; Fathi et al., 2017). However, a few studies also indicated insignificant effects of milk and fermented milk products on lipid profile (St-Onge et al., 2002; Maki et al., 2015; Ostadrahimi et al., 2015).

Again, the beneficial effects of kefir on glucose level may be related to an alteration in the intestinal microbiota. Because reduced intestinal permeability could reduce oxidative stress and low-grade chronic inflammation (Gomes et al., 2014; Rosa et al., 2017), the function of insulin receptors could be restored, leading to a better control of blood glucose (Rosa et al., 2017). Nevertheless, many studies have demonstrated that milk, especially milk proteins and bioactive peptides, has glucose regulatory properties (reviewed in Nongonierma and FitzGerald, 2015; El-Sayed et al., 2016). The results from El-Sayed et al. (2016) suggest that milk protein, especially milk protein hydrolysate, significantly reduces the concentration of plasma glucose and could be a good source of anti-diabetic agents. The suggested mechanism proposes an insulinotropic activity, incretin secretagogue action, as well as activity on different metabolic enzymes involved in the regulation of serum glucose (Lacroix and Li-Chan, 2014). A similar influence of kefir and milk on glucose levels in the present study favors the later explanation. The intakes of carbohydrates, fats, proteins, and daily energy intake were unchanged during both interventions and therefore cannot explain the observed differences in the glucose level.

Several mechanisms have been proposed to justify the potential of kefir supplementation in reducing cholesterol levels. Kefir supplementation increases the pro-

Table 6. Mood (affect) and appetite after kefir and milk supplementation ${ }^{1}$

\begin{tabular}{|c|c|c|c|c|c|c|c|}
\hline Item & \multicolumn{3}{|c|}{ Kefir intake } & \multicolumn{3}{|c|}{ Milk intake } & $P$-value ${ }^{2}$ \\
\hline Positive affect & $33.4 \pm 4.8$ & $34.3 \pm 4.3$ & 0.033 & $34.2 \pm 5.9$ & $31.9 \pm 6.9$ & 0.032 & 0.014 \\
\hline Appetite & $15.9 \pm 3.9$ & $15.5 \pm 3.7$ & 0.24 & $16.2 \pm 1.9$ & $16.2 \pm 2.0$ & 1.00 & 0.377 \\
\hline
\end{tabular}

${ }^{1}$ Values are presented as means \pm SD.

${ }^{2} P$-value denotes comparison of mean changes between the 2 groups using an independent $t$-test.

${ }^{3} P$-value denotes difference within the 2 groups using a paired $t$-test. 
duction of short-chain fatty acids, which are believed to reduce the production of cholesterol levels either by inhibiting hydroxymethylglutaryl CoA reductase or by redistributing plasma cholesterol to the liver (St-Onge et al., 2002; Wang et al., 2009; Arora et al., 2011). Another possible mechanism involves the production of bile salt hydrolase enzyme by bacteria and yeasts present in kefir grains, the enzyme that catalyzes the deconjugation of bile acids and increases their excretion in the feces, therefore helping to lower cholesterol levels (Liu et al., 2012). Several nutrients and bioactive compounds present in milk have been hypothesized to have desirable effects on serum lipid levels (reviewed in Nongonierma and FitzGerald, 2015). For example, high calcium intake from dairy products, present in similar amounts in kefir and milk used in our study (Table 1), has been shown to lower serum total cholesterol and LDL levels in humans (Soerensen et al., 2014), probably by increasing fecal fat excretion (Christensen et al., 2009).

In the present study, we found no significant changes in anthropometric variables after kefir and milk supplementation. The lack of any significant effect on BW is similar to previous reports (Ostadrahimi et al., 2015) and was not expected in such a short intervention.

Finally, based on our results, kefir supplementation significantly increased positive mood. Steenbergen et al. (2015) provided the first evidence that the intake of probiotics may help to reduce sad mood in healthy individuals. In a recent study, it has been also shown that kefir could be used as a diet to prevent depression, anxiety, cognitive impairment, and an available natural therapy for patients suffering from nicotine-induced anxiety and depression as seen in the animal model (Noori et al., 2014). The mechanism includes the action of tryptophan (serotonin precursor) as an essential AA abundant in kefir (Garrote et al., 2001; Otles and Cagindi, 2003). Therefore, kefir as a food supplement with a high level of tryptophan can alter serotonin level and thus can be effective in enhancing positive moods. However, further studies are warranted.

We must stress that the present study has certain limitations. The participants were allowed to remain in a "free-living" environment, so the results are based on participant's motivation and compliance. The participants were instructed to omit dairy products other than the supplement, but were otherwise asked to keep their normal diet. A 24-h recall was checked 3 times during the kefir and milk supplementation period to control that they indeed followed the instructions. It is noteworthy, however, the participants were living in a free-living environment and we cannot fully exclude the possibility that they did not include all information. Also, the information of the exact bacterial profile of our product is not exhaustive, which could be relevant considering the evidence that probiotic effects are species and even strain specific.

\section{CONCLUSIONS}

Our study is the first showing that kefir supplementation causes an improvement in serum zonulin levels, and positive effects, compared with milk supplementation, and yet similar improvement in lipid profile and serum glucose levels compared with milk supplementation in asymptomatic overweight adults.

\section{ACKNOWLEDGMENTS}

The study was co-financed by the Republic of Slovenia Ministry of Education, Science and sport and European Union from European Social Funds. This work was also supported by the Slovenian Research Agency (Programme P1-0386 and research project J3-8209). The authors thank all the subjects for volunteering in this study, and students and nurses of the Faculty of Health Sciences. AP, ZJP, TV, and SK designed the study; ZJP, AP, SK, and MČB conducted the study and collected and analyzed data; AP wrote the initial draft of the manuscript and all authors contributed to the editing and review of this manuscript. All authors read and approved the final manuscript. Tinkara Vardjan is an employee of Kele \& Kele. T. Vardjan is specialized in the analysis and the production of kefir from kefir grains and was invited to help with the design of the study. As she is an employee of the company whose kefir drink was used in the intervention, she was involved only in the design of the study but was then restricted from data analysis and interpretation. The authors have not stated any conflicts of interest.

\section{REFERENCES}

Adiloğlu, A. K., N. Gönülateş, M. Işler, and A. Senol. 2013. The effect of kefir consumption on human immune system: A cytokine study. Mikrobiyol. Bul. 47:273-281. https://doi.org/10.5578/mb.4709.

Alberti, K. G., R. H. Eckel, S. M. Grundy, P. Z. Zimmet, J. I. Cleeman, K. A. Donato, J. C. Fruchart, W. P. James, C. M. Loria, and S. C. Smith Jr. 2009. Harmonizing the metabolic syndrome: A joint interim statement of the International Diabetes Federation Task Force on Epidemiology and Prevention; National Heart, Lung, and Blood Institute; American Heart Association; World Heart Federation; International Atherosclerosis Society; and International Association for the Study of Obesity. Circulation 120:1640-1645. https://doi.org/10.1161/CIRCULATIONAHA.109.192644.

Arora, T., R. Sharma, and G. Frost. 2011. Propionate. Anti-obesity and satiety enhancing factor? Appetite 56:511-515. https://doi .org/10.1016/j.appet.2011.01.016.

Arrieta, M. C., L. Bistritz, and J. B. Meddings. 2006. Alterations in intestinal permeability. Gut 55:1512-1520. https://doi.org/10 .1136 /gut.2005.085373.

Boroni Moreira, A. P., and R. de Cássia Gonçalves Alfenas. 2012. The influence of endotoxemia on the molecular mechanisms of insulin 
resistance. Nutr. Hosp. 27:382-390. https://doi.org/10.1590/S0212 $-16112012000200007$.

Boulangé, C. L., A. L. Neves, J. Chilloux, J. K. Nicholson, and M. E. Dumas. 2016. Impact of the gut microbiota on inflammation, obesity, and metabolic disease. Genome Med. 8:42. https://doi.org/10 $.1186 / \mathrm{s} 13073-016-0303-2$.

Cani, P. D., R. Bibiloni, C. Knauf, A. Waget, A. M. Neyrinck, N. M. Delzenne, and R. Burcelin. 2008. Changes in gut microbiota control metabolic endotoxemia-induced inflammation in high-fat diet-induced obesity and diabetes in mice. Diabetes 57:1470-1481. https://doi.org/10.2337/db07-1403.

Chassaing, B., and A. T. Gewirtz. 2014. Gut microbiota, low-grade inflammation, and metabolic syndrome. Toxicol. Pathol. 42:49-53. https://doi.org/10.1177/0192623313508481.

Christensen, R., J. K. Lorenzen, C. R. Svith, E. M. Bartels, E. L. Melanson, W. H. Saris, A. Tremblay, and A. Astrup. 2009. Effect of calcium from dairy and dietary supplements on faecal fat excretion: A meta-analysis of randomized controlled trials. Obes. Rev. 10:475-486. https://doi.org/10.1111/j.1467-789X.2009.00599.x.

Ejtahed, H.-S., P. Angoorani, A.-R. Soroush, R. Atlasi, S. HasaniRanjbar, A. M. Mortazavian, and B. Larijani. 2019. Probiotics supplementation for the obesity management; A systematic review of animal studies and clinical trials. J. Funct. Foods 52:228-242. https://doi.org/10.1016/j.jff.2018.10.039.

El-Sayed, M., S. Awad, A. Wahba, A. El-Attar, M. Yousef, and M. Zedan. 2016. In vivo anti-diabetic and biological activities of milk protein and milk 1 protein hydrolyaste. J. Adv. Dairy Res. 4:1.

Everard, A., and P. D. Cani. 2013. Diabetes, obesity and gut microbiota. Best Pract. Res. Clin. Gastroenterol. 27:73-83. https://doi .org/10.1016/j.bpg.2013.03.007.

Fathi, Y., N. Ghodrati, M. J. Zibaeenezhad, and S. Faghih. 2017. Kefir drink causes a significant yet similar improvement in serum lipid profile, compared with low-fat milk, in a dairy-rich diet in overweight or obese premenopausal women: A randomized controlled trial. J. Clin. Lipidol. 11:136-146. https://doi.org/10.1016/j.jacl 2016.10.016.

Festi, D., R. Schiumerini, L. H. Eusebi, G. Marasco, M. Taddia, and A. Colecchia. 2014. Gut microbiota and metabolic syndrome. World J. Gastroenterol. 20:16079-16094. https://doi.org/10.3748/wjg .v20.i43.16079.

Gao, J., G. Ding, Q. Li, L. Gong, J. Huang, and Y. Sang. 2019. Tibet kefir milk decreases fat deposition by regulating the gut microbiota and gene expression of Lpl and Angptl4 in high fat diet-fed rats. Food Res. Int. 121:278-287. https://doi.org/10.1016/j.foodres 2019.03.029.

Garrote, G. L., A. G. Abraham, and G. L. De Antoni. 2001. Chemical and microbiological characterisation of kefir grains. J. Dairy Res. 68:639-652. https://doi.org/10.1017/S0022029901005210.

Genser, L., D. Aguanno, H. A. Soula, L. Dong, L. Trystram, K. Assmann, J. E. Salem, J. C. Vaillant, J. M. Oppert, F. Laugerette, M. C. Michalski, P. Wind, M. Rousset, E. Brot-Laroche, A. Leturque, K. Clément, S. Thenet, and C. Poitou. 2018. Increased jejunal permeability in human obesity is revealed by a lipid challenge and is linked to inflammation and type 2 diabetes. J. Pathol. 246:217230. https://doi.org/10.1002/path.5134.

Gérard, P. 2016. Gut microbiota and obesity. Cell. Mol. Life Sci. 73:147-162. https://doi.org/10.1007/s00018-015-2061-5.

Gomes, A. C., A. A. Bueno, R. G. de Souza, and J. F. Mota. 2014. Gut microbiota, probiotics and diabetes. Nutr. J. 13:60. https:// doi.org/10.1186/1475-2891-13-60.

Griffiths, E. A., L. C. Duffy, F. L. Schanbacher, H. Qiao, D. Dryja, A. Leavens, J. Rossman, G. Rich, D. Dirienzo, and P. L. Ogra. 2004. In vivo effects of bifidobacteria and lactoferrin on gut endotoxin concentration and mucosal immunity in Balb/c mice. Dig. Dis. Sci. 49:579-589. https://doi.org/10.1023/B:DDAS.0000026302 .92898.ae.

Hadisaputro, S., R. R. Djokomoeljanto, Judiono, and M. H. Soesatyo. 2012. The effects of oral plain kefir supplementation on proinflammatory cytokine properties of the hyperglycemia Wistar rats induced by streptozotocin. Acta Med. Indones. 44:100-104.
Jayashree, B., Y. S. Bibin, D. Prabhu, C. S. Shanthirani, K. Gokulakrishnan, B. S. Lakshmi, V. Mohan, and M. Balasubramanyam. 2014. Increased circulatory levels of lipopolysaccharide (LPS) and zonulin signify novel biomarkers of proinflammation in patients with type 2 diabetes. Mol. Cell. Biochem. 388:203-210. https:// doi.org/10.1007/s11010-013-1911-4.

Kim, D. H., H. Kim, D. Jeong, I. B. Kang, J. W. Chon, H. S. Kim, K. Y. Song, and K. H. Seo. 2017. Kefir alleviates obesity and hepatic steatosis in high-fat diet-fed mice by modulation of gut microbiota and mycobiota: Targeted and untargeted community analysis with correlation of biomarkers. J. Nutr. Biochem. 44:35-43. https://doi .org/10.1016/j.jnutbio.2017.02.014.

Lacroix, I. M., and E. C. Li-Chan. 2014. Overview of food products and dietary constituents with antidiabetic properties and their putative mechanisms of action: A natural approach to complement pharmacotherapy in the management of diabetes. Mol. Nutr. Food Res. 58:61-78. https://doi.org/10.1002/mnfr.201300223.

Lee, M. Y., K. S. Ahn, O. K. Kwon, M. J. Kim, M. K. Kim, I. Y. Lee, S. R. Oh, and H. K. Lee. 2007. Anti-inflammatory and antiallergic effects of kefir in a mouse asthma model. Immunobiology 212:647-654. https://doi.org/10.1016/j.imbio.2007.05.004.

Li, X., J. Yin, Y. Zhu, X. Wang, X. Hu, W. Bao, Y. Huang, L. Chen, S. Chen, W. Yang, Z. Shan, and L. Liu. 2018. Effects of whole milk supplementation on gut microbiota and cardiometabolic biomarkers in subjects with and without lactose malabsorption. Nutrients 10:1403. https://doi.org/10.3390/nu10101403.

Liu, H., Y. H. Xie, L. X. Xiong, R. T. Dong, C. L. Pan, G. X. Teng, and H. X. Zhang. 2012. Effect mechanism of cholesterol lowering by Kluyveromyces from Tibetan kefir. Adv. Mat. Res. 343:12901298.

Maki, K. C., K. M. Nieman, A. L. Schild, V. N. Kaden, A. L. Lawless, K. M. Kelley, and T. M. Rains. 2015. Sugar-sweetened product consumption alters glucose homeostasis compared with dairy product consumption in men and women at risk of type 2 diabetes mellitus. J. Nutr. 145:459-466. https://doi.org/10.3945/jn.114 .204503 .

Moreno-Navarrete, J. M., M. Sabater, F. Ortega, W. Ricart, and J. M. Fernández-Real. 2012. Circulating zonulin, a marker of intestinal permeability, is increased in association with obesity-associated insulin resistance. PLoS One 7:e37160. https://doi.org/10.1371/ journal.pone.0037160.

Nongonierma, A. B., and R. J. FitzGerald. 2015. The scientific evidence for the role of milk protein-derived bioactive peptides in humans. J. Funct. Foods 17:640-656. https://doi.org/10.1016/j.jff .2015.06.021.

Noori, N., M. Y. Bangash, M. Motaghinejad, P. Hosseini, and B. Noudoost. 2014. Kefir protective effects against nicotine cessationinduced anxiety and cognition impairments in rats. Adv. Biomed. Res. 3:251. https://doi.org/10.4103/2277-9175.146377.

Norris, G. H., C. Jiang, J. Ryan, C. M. Porter, and C. N. Blesso. 2016 Milk sphingomyelin improves lipid metabolism and alters gut microbiota in high fat diet-fed mice. J. Nutr. Biochem. 30:93-101. https://doi.org/10.1016/j.jnutbio.2015.12.003.

Ostadrahimi, A., A. Taghizadeh, M. Mobasseri, N. Farrin, L. Payahoo, Z. Beyramalipoor Gheshlaghi, and M. Vahedjabbari. 2015. Effect of probiotic fermented milk (kefir) on glycemic control and lipid profile in type 2 diabetic patients: A randomized double-blind placebo-controlled clinical trial. Iran. J. Public Health 44:228-237.

Otles, S., and O. Cagindi. 2003. Kefir: A probiotic dairy-composition, nutritional and therapeutic aspects. Pak. J. Nutr. 2:54-59. https:/ /doi.org/10.3923/pjn.2003.54.59.

Ouchi, N., J. L. Parker, J. J. Lugus, and K. Walsh. 2011. Adipokines in inflammation and metabolic disease. Nat. Rev. Immunol. 11:85-97. https://doi.org/10.1038/nri2921.

Pereira, M. A., D. R. Jacobs Jr., L. Van Horn, M. L. Slattery, A. I. Kartashov, and D. S. Ludwig. 2002. Dairy consumption, obesity, and the insulin resistance syndrome in young adults: The CARDIA Study. JAMA 287:2081-2089. https://doi.org/10.1001/jama .287.16.2081. 
Pimenta, F. S., M. Luaces-Regueira, A. M. Ton, B. P. Campagnaro, M. Campos-Toimil, T. M. Pereira, and E. C. Vasquez. 2018. Mechanisms of action of kefir in chronic cardiovascular and metabolic diseases. Cell. Physiol. Biochem. 48:1901-1914. https://doi.org/10 $.1159 / 000492511$.

Rios, A. C., P. K. Maurya, M. Pedrini, M. Zeni-Graiff, E. Asevedo, R. B. Mansur, A. Wieck, R. Grassi-Oliveira, R. S. McIntyre, M. A. F. Hayashi, and E. Brietzke. 2017. Microbiota abnormalities and the therapeutic potential of probiotics in the treatment of mood disorders. Rev. Neurosci. 28:739-749. https://doi.org/10.1515/revneuro $-2017-0001$.

Rodrigues, K. L., L. R. Caputo, J. C. Carvalho, J. Evangelista, and J. M. Schneedorf. 2005. Antimicrobial and healing activity of kefir and kefiran extract. Int. J. Antimicrob. Agents 25:404-408. https: //doi.org/10.1016/j.ijantimicag.2004.09.020.

Rosa, D. D., M. M. S. Dias, L. M. Grześkowiak, S. A. Reis, L. L. Conceição, and M. D. C. G. Peluzio. 2017. Milk kefir: nutritional, microbiological and health benefits. Nutr. Res. Rev. 30:82-96. https: //doi.org/10.1017/S0954422416000275.

Rosa, D. D., Ł. M. Grześkowiak, C. L. Ferreira, A. C. Fonseca, S. A. Reis, M. M. Dias, N. P. Siqueira, L. L. Silva, C. A. Neves, L. L. Oliveira, A. B. Machado, and C. Peluzio. 2016. Kefir reduces insulin resistance and inflammatory cytokine expression in an animal model of metabolic syndrome. Food Funct. 7:3390-3401. https:// doi.org/10.1039/C6FO00339G.

Sapone, A., L. de Magistris, M. Pietzak, M. G. Clemente, A. Tripathi, F. Cucca, R. Lampis, D. Kryszak, M. Cartenì, M. Generoso, D. Iafusco, F. Prisco, F. Laghi, G. Riegler, R. Carratu, D. Counts, and A. Fasano. 2006. Zonulin upregulation is associated with increased gut permeability in subjects with type 1 diabetes and their relatives. Diabetes 55:1443-1449. https://doi.org/10.2337/db05-1593.

Scheffler, L., A. Crane, H. Heyne, A. Tönjes, D. Schleinitz, C. H. Ihling, M. Stumvoll, R. Freire, M. Fiorentino, A. Fasano, P. Kovacs, and J. T. Heiker. 2018. Widely used commercial ELISA does not detect precursor of haptoglobin2, but recognizes properdin as a potential second member of the zonulin family. Front Endocrinol (Lausanne). 9:22. https://doi.org/10.3389/fendo.2018.00022.

Scherer, P. E., and J. A. Hill. 2016. Obesity, diabetes, and cardiovascular diseases: A compendium. Circ. Res. 118:1703-1705. https:// doi.org/10.1161/CIRCRESAHA.116.308999.

Sepple, C. P., and N. W. Read. 1989. Gastrointestinal correlates of the development of hunger in man. Appetite 13:183-191. https://doi .org/10.1016/0195-6663(89)90011-1.

Soerensen, K. V., T. K. Thorning, A. Astrup, M. Kristensen, and J. K. Lorenzen. 2014. Effect of dairy calcium from cheese and milk on fecal fat excretion, blood lipids, and appetite in young men. Am. J. Clin. Nutr. 99:984-991. https://doi.org/10.3945/ajcn.113.077735.

St-Onge, M. P., E. R. Farnworth, T. Savard, D. Chabot, A. Mafu, and P. J. Jones. 2002. Kefir consumption does not alter plasma lipid levels or cholesterol fractional synthesis rates relative to milk in hyperlipidemic men: a randomized controlled trial. BMC Complement. Altern. Med. 2:1. https://doi.org/10.1186/1472-6882-2-1.
Steenbergen, L., R. Sellaro, S. van Hemert, J. A. Bosch, and L. S. Colzato. 2015. A randomized controlled trial to test the effect of multispecies probiotics on cognitive reactivity to sad mood. Brain Behav. Immun. 48:258-264. https://doi.org/10.1016/j.bbi.2015.04 .003 .

Sun, Y., W. Geng, Y. Pan, J. Wang, P. Xiao, and Y. Wang. 2019. Supplementation with Lactobacillus kefiranofaciens ZW3 from Tibetan Kefir improves depression-like behavior in stressed mice by modulating the gut microbiota. Food Funct. 10:925-937. https:// doi.org/10.1039/C8FO02096E.

Talley, N. J., G. J. Holtmann, M. Jones, N. A. Koloski, M. M. Walker, G. Burns, M. D. E. Potter, A. Shah, and S. Keely. 2019. Zonulin in serum as a biomarker fails to identify the IBS, functional dyspepsia and non-coeliac wheat sensitivity. Gut gutjnl-2019-318664. https://doi.org/10.1136/gutjnl-2019-318664.

van Hemert, S., J. Verwer, and B. Schütz. 2013. Clinical studies evaluating effects of probiotics on parameters of intestinal barrier function. Adv. Microbiol. 3:212-222. https://doi.org/10.4236/aim.2013 .32032 .

Vardjan, T., P. M. Lorbeg, and A. Č. Majhenič. 2018. Stability of prevailing lactobacilli and yeasts in kefir grains and kefir beverages during ten weeks of propagation. Int. J. Dairy Technol. 71:51-60. https://doi.org/10.1111/1471-0307.12463.

Vardjan, T., P. Mohar Lorbeg, I. Rogelj, and A. Čanžek Majhenič. 2013. Characterization and stability of lactobacilli and yeast microbiota in kefir grains. J. Dairy Sci. 96:2729-2736. https://doi .org/10.3168/jds.2012-5829.

Vinderola, C. G., J. Duarte, D. Thangavel, G. Perdigón, E. Farnworth, and C. Matar. 2005. Immunomodulating capacity of kefir. J. Dairy Res. 72:195-202. https://doi.org/10.1017/S0022029905000828.

Wang, W., S. Uzzau, S. E. Goldblum, and A. Fasano. 2000. Human zonulin, a potential modulator of intestinal tight junctions. J. Cell Sci. 113:4435-4440.

Wang, Y., N. Xu, A. Xi, Z. Ahmed, B. Zhang, and X. Bai. 2009. Effects of Lactobacillus plantarum MA2 isolated from Tibet kefir on lipid metabolism and intestinal microflora of rats fed on highcholesterol diet. Appl. Microbiol. Biotechnol. 84:341-347. https:// doi.org/10.1007/s00253-009-2012-x.

Watson, D., L. A. Clark, and A. Tellegen. 1988. Development and validation of brief measures of positive and negative affect: The PANAS scales. J. Pers. Soc. Psychol. 54:1063-1070. https://doi .org/10.1037/0022-3514.54.6.1063.

Zak-Gołąb, A., P. Kocełak, M. Aptekorz, M. Zientara, L. Juszczyk, G. Martirosian, J. Chudek, and M. Olszanecka-Glinianowicz. 2013. Gut microbiota, microinflammation, metabolic profile, and zonulin concentration in obese and normal weight subjects. Int. J. Endocrinol. 2013:674106. https://doi.org/10.1155/2013/674106.

Zimmet, P. Z., and K. G. Alberti. 1997. The changing face of macrovascular disease in non-insulin-dependent diabetes mellitus: An epidemic in progress. Lancet 350:S1-S14. https://doi.org/10.1016/ S0140-6736(97)90020-9. 